Radial and Nonradial Pulsations as Probes of Stellar Physics

ASP Conference Series, Vol. 259, 2002

C. Aerts, T.R. Bedding, \& J. Christensen-Dalsgaard, eds.

\title{
Seismic Tests of Theoretical Models of HR 1217
}

\author{
M.S. Cunha ${ }^{1,2}$, J.M.M.B. Fernandes ${ }^{3}$, M.J.P.F.G. Monteiro ${ }^{1,4}$ \\ 1 Centro de Astrofísica da Universidade do Porto,Portugal \\ ${ }^{2}$ Instituto Superior da Maia, Portugal \\ ${ }^{3}$ Observatório Astronómico da Universidade de Coimbra, Portugal \\ ${ }^{4}$ Departamento de Matemática Aplicada da Faculdade de Ciencias, \\ Universidade do Porto, Portugal
}

\section{Introduction}

A way in which we might learn about the physics of the interior of roAp stars is by studying their oscillations. To that aim we have initiated a study in order to investigate what additional constraints might be imposed to the physics of the interior of roAp stars from the study of their oscillation spectra. We have chosen to start this work by studying the well known multi-periodic roAp star HR 1217 . The observed data used for HR1217 were $L / L_{\odot}=7.8 \pm 0.7$ and $\mathrm{T}_{\text {eff }}=7400 \pm 100$ $\mathrm{K}$, from Matthews et al. (1999), $Z_{s}=0.009$ estimated from Ryabchikova et al. (1997), and $\Delta \nu=67.91 \pm 0.12 \mu \mathrm{Hz}$ from Kurtz et al. (1989).

The seismic data must be consistent with all other observables for the star. However, there are several aspects of the structure of roAp stars that have to be described by parameters. Cunha et al. (2001) have shown that at constant mass, the effect produced in the evolutionary tracks of models appropriate to roAp stars by a change in the abundance of heavy elements is much greater than the effect produced by a change in other parameters, like helium abundance, mixing length and overshooting in the convective core. Consequently, here we turn our attention to the dependence of the oscillations on the abundance of heavy elements. Note that, although the abundance of heavy elements at the surface of HR1217 might be determined from the observations, the latter is not necessarily a good representation of the abundance in the interior of the star.

\section{Results}

We evolved stellar models characterised by different pairs of parameters $(M, Z)$, where $M$ is the mass of the model and $Z$ is the abundance of heavy elements, from the ZAMS, using the CESAM code (Morel, 1997). With $Z$ fixed, the mass was chosen in such a way as to assure that the corresponding model reproduced, at some point during the main sequence, the observed effective temperature and luminosity of HR1217. The oscillation spectra of these models, at the age when they best reproduced HR1217, were calculated by means of a linear adiabatic oscillation code, and an asymptotic relation was used to relate the large separations to the corresponding masses.

In Fig. 1 we plot the ratio between the mass of our models and the mass that the star should have, considering the observables available $\left(\mathrm{T}_{\text {eff }}, L, \Delta \nu\right)$. 
Moreover, the values of this ratio that are allowed by the errors associated with the effective temperature and luminosity are marked by the region shadowed by squares. The region shadowed by lines shows values that would still be consistent with the observations if the effective temperature were allowed to vary between 7200 and $7500 \mathrm{~K}$ (to include the value given by Ryabchikova et al., 1997).

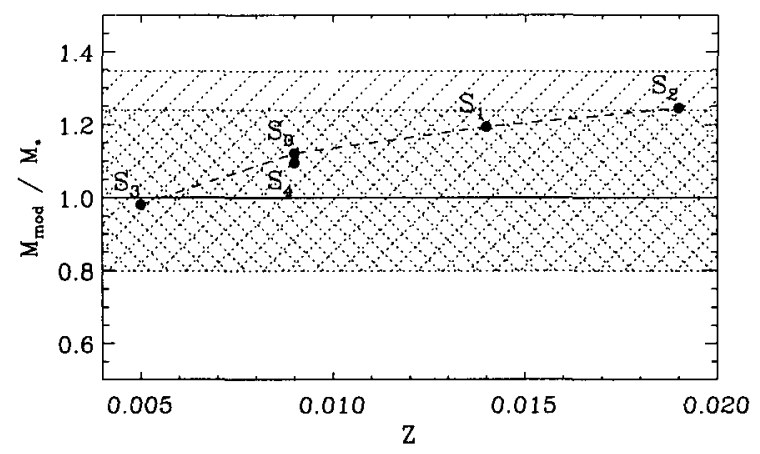

Figure 1. Ratio between the mass of our models and the one based on data. The pairs $(M, Z)$ used were: $\mathrm{S}_{0}(1.5,0.009) ; \mathrm{S}_{1}(1.6,0.014)$; $\mathrm{S}_{2}(1.67,0.019) ; \mathrm{S}_{3}(1.37,0.005) ; \mathrm{S}_{4}$-same as $\mathrm{S}_{0}$ but with $T-\tau$ relation defined by Gautschy et al. (1998), instead of an Eddington atmosphere.

The model that best satisfies the observations is $S_{3}$. However, this model is not a plausible one, since its value of $Z$ (Fig. 1) is characteristic of stars in the limit between populations I and II. Even if not so close to reproducing the observations, all the other models lie within the error bars. However, if found to be systematic, when other roAp stars are considered, these results might be indicative of an underabundance of heavy elements in the interior of roAp stars.

The introduction of a temperature inversion, as suggested by Gautschy et al. (1998), does not seem to influence the results in a significant way.

Acknowledgments. This work was supported by FCT-Portugal through the grant PD/18893/98 and the projects PESO/P/PRO15128/1999 and POCTI/1999/FIS/34549.

\section{References}

Cunha, M.S., Fernandes, J.M.M.B., Monteiro, M.J.P.F.G. 2001, in ASP Conf. Ser., Observed HR Diagrams and Stellar Evolution, eds. J. Fernandes \& T. Lejeune, (San Francisco ASP), in press

Gautschy, A., Saio, H., \& Harzenmoser, H. 1998, MNRAS, 301, 31

Kurtz, D.W., et al. 1989, MNRAS, 240, 881

Matthews, J.M., Kurtz, D.W., \& Martinez, P. 1999, ApJ, 511, 422

Morel, P. 1997, A\&AS, 124, 597

Ryabchikova, T.A., et al. 1997, A\&A, 327, 1137

Shibahashi, H. \& Saio, H. 1985, PASJ, 37, 245 




Mike Breger is singing a song for Steve and Leslie Kawaler. Doris Baker is wondering when he will stop... 